nephritis. It was effective in 6 cases $(43 \%)$ and half of them were from lupus nephritis. There was significant improvement in renal function in 3 cases of lupus nephritis out of the 4 cases. Anticoagulant therapy was started in the early stage from the onset and warfarin after heparin was adminstered more than 6 months in these successful cases. From our standpoint of view, it is effective to start anticoagulant therapy in the early stage of lupus nephritis. It is helpful to maintain the kidney function and to protect the kidney from the tissue damage. Lupus nephritis is a kind disease which benefits by anticoagulant therapy.

\title{
III. Lupus Erythematosus (Supplementary) Treatment and Prognosis of Lupus Nephritis
}

\author{
Hiroshi Hashimoto, MD and Yuichi Shiokawa, MD \\ Division of Rheumatology, Department of Internal Medicine, \\ School of Medicine, Juntendo University, Tokyo.
}

The purpose of this work is (1) to study the long-term prognosis of SLE patients who received renal biopsies and who started therapy with various types of treatment, and (2) to try to set up a therapeutic regimen for lupus nephritis. It also discusses whether the treatments alter the natural course of lupus nephritis or not.

\section{MATERIAL AND METHOD}

Renal tissue from 110 SLE patients diagnosed as having satisfied ARA criteria for SLE were examined histologically. They were classified into 6 types; 1. minimal change (MC) (42 cases), 2. focal proliferative GN (FGN) (15 cases), 3. diffuse proliferative GN (DPGN) (16 cases), 4. membranous GN (MGN) (15 cases), 5. membranoproliferative GN (MPGN) (18 cases), 6. advanced stage (4 cases). The types of treatment were classified as follows: 1. low doses of steroids, 2. high doses of steroids, 3. combined therapy of steroids and cytostatics. SLE patients treated with high doses of steroids included the patients with MC and FGN treated with over $30 \mathrm{mg} /$ day of prednisone, and the patients with DPGN, MGN and advanced stage treated with over $40 \mathrm{mg} /$ day. Cyclophosphamide, azathioprine and 6 mercaptoprine were used as cytostatics. Survivalship of lupus nephritis after starting therapy with these types of treatment was evaluated by the life table method. The differences of the responses of steroids for clinical and immunological findings among the histological types of nephritis after the start of treatment with $40 \mathrm{mg} /$ day were studied. In SLE patients with DPGN, MGN and MPGN, the changes in clinical and immunological findings during the treatment were also studied. Relationship between the treatment and transformation of glomerular lesion of lupus nephritis using rebiopsy or autopsy specimen was studied. 


\section{RESULTS AND CONCLUSION}

There were differences among the histological types of lupus nephritis with respect to survivalship and the responses of steroids for clinical and immunological findings. The patients with FGN, DPGN, and MPGN who were treated with combined therapy of steroids and cytostatics had a better prognosis than the patients treated with steroids alone. In the changes in laboratory findings following treatment with high doses of steroids in SLE patients with DPGN, MGN and MPGN, persistent proteinuria under $3.5 \mathrm{~g} /$ day 3 and 6 months after treatment. High titer of anti DNA antibodies and low complement levels also improved significantly after the start of treatment. However, these complement levels had a tendency to get worse again by the 24 th month of treatment. On the other hand, low complement levels showed significant improvement at 12 and 24 months after the start of treatment with combined therapy in DPGN, MGN and MPGN. Normalization of complement levels by combined therapy seems to be associated with stabilization or diminution of lupus nephritis.

The prognosis of the patients with FGN and DPGN treated with high doses of steroids was better than that of patients treated with low doses of steroids. However, the patients with MGN treated with high doses of steroids had a poorer prognosis than the patients treated with low doses of steroids or combined therapy. Within 6 months from the start of treatment with high doses of steroids, 3 patients died from causes unrelated to SLE. For SLE patients with advanced stage, who had the poorest prognosis of all the 6 types of lupus nephritis, steroids therapy seemed not to be advisable.

High doses of steroids or combined therapy of cytostatics and steroids could not prevent progression of glomerular lesions from MC or FGN to DPGN, MGN or MPGN, and we do not think that such treatment alters the long-term prognosis of lupus nephritis but that it does have the effect of prolonging the patients' lives.

\title{
III. Lupus Erythmatosus Supqiementary: A Study on Lupus Nephritis. Serial Renal Biopsies Especially in the Patients with Non-Renal SLE
}

\author{
Fuyuhiko Higashi, MD and Ippei Fujimori, MD
}

Department of Internal Medicine, Kawasaki Municipal Hospital

Comparative studies among renal histopathological findings, clinical manifestations (ARA preliminary criteria) and the results of immunoserological examinations were made on 58 cases of patients with
SLE between 1972-1978.

Of 20 cases to whom serial renal biopsies were performed, the relation between the dosage of prednisolone and the course of renal histological changes was 